\title{
Efeito do processamento do grão sobre a população de protozoários ruminais de ovinos Santa Inês
}

Oliveira Abrão, F. ${ }^{\circledR}$; Oliveira Santos, E. ${ }^{\text {; }}$ Dijkstra, D. ' ; Fabino Neto, R. ${ }^{\text {; }}$; Curcino Batista, L.H. ${ }^{\text {; }}$ Robson Duarte, E. ${ }^{2}$

'Instituto Federal Goiano. Goias. Brasil.

2Universidade Federal de Minas Gerais. Minas Gerais, Brasil.

\section{PALAVRAS CHAVE ADICIONAIS}

Borregas.

Entodinium spp.

Fluido ruminal.

Milho.

Sorgo.

\section{RESUMO}

Objetivou-se com a presente pesquisa caracterizar a população de protozoários do rúmen de ovinos alimentados com milho e sorgo submetidos a diferentes tipos de processamento. Em um delineamento inteiramente casualizado foram avaliados quatro tratamentos com sete repetições. As borregas foram submetidas a uma dieta com relação volumoso:concentrado de 40:60 na matéria seca. O concentrado foi composto pelos alimentos testados: milho grão seco, milho grão reidratado, sorgo grão seco e sorgo grão reidratado. O volumoso da dieta foi o feno de capim bermudas (Cynodom dactylon). Os animais foram confinados, totalizando um período experimental de 60 dias. No último dia do experimento, foi realizada a coleta por punção do fluido ruminal com auxílio de um cateter e de seringa estéril para análises macroscópicas e físico-químicas do fluido ruminal. As concentrações de protozoários foram submetidas ao teste de Kruskall-Wallis e as frequências dos gêneros foram comparadas pelo teste do Qui-quadrado. Verificou-se que o processamento do grão em dieta mista não interferiu na concentração de médios e grandes protozoários, no entanto a concentração de pequenos protozoários foi superior quando a fonte de concentrado foi o sorgo grão reidratado e ensilado e inferior em dietas constituídas por milho grão seco. O gênero Entodinium foi o mais frequente em todos grupos avaliados.

\section{Effect of processing the grain on ruminal protozoa population of Santa Inês sheep}

\section{SUMMARY}

\section{ADDITIONAL KEYWORDS}

Ewe lambs.

Entodinium spp.

Rumen fluid.

Corn.

Sorghum.

INFORMATION

Cronología del artículo.

Recibido/Received: 15.06.2017

Aceptado/Accepted: 22.05 .2018

On-line: 15.10 .2018

Correspondencia a los autores/Contact e-mail:

flavia.abrao@ifgoiano.edu.br
The aim in this study was to characterize the population of ruminal protozoa of sheep fed with corn four treatments with seven repetitions. The female lambs were subjected to a diet about forage:concentrate 40:60 on dry matter. The concentrate formulated was composed of the foods tested: dry grain corn, grain corn rehydrated, dry grain sorghum and sorghum grain rehydrated. The forage of the diet was the Bermuda grass hay (Cynodom dactylon). The animals were confined totaling a trial period of 60 days. In the last day of the experiment, we sampled the ruminal fluid by puncture using collection, with the aid of a catheter and a sterile syringe, for subsequent execution of macroscopic and physico-chemical analyses of the ruminal fluid. The protozoa concentrations were subjected to the test Kruskall-Wallis and of the genus frequencies were compared by the test $\mathrm{Chi}$-Square. The grain processing in mixed diet did not interfere with the concentration of medium and large protozoa, however the small protozoa concentration was higher when the lambs was fed silage of rehydrated sorghum grain and lower when fed dry grain corn. The Entodinium spp. was the most frequent genus of ciliates for all treatments. and sorghum subjected to different types of processing. In a completely randomized design we evaluated

\section{INTRODUÇÃO}

Os ruminantes constituem um grupo de animais com diferentes características na digestão de alimentos, os nutrientes ingeridos sofrem a ação de microrganismos para serem absorvidos posteriormente. O ecossistema ruminal desses animais é composto principalmente por bactérias, fungos e protozoários, que desempenham funções bioquímicas e fisiológicas importantes para os ruminantes, pois produzem enzimas capazes de degradar os principais carboidratos das plantas, fornecendo energia ao hospedeiro em uma relação mutualística (Reis, 2015; Wlodarski, 2017). 
A presença de protozoários no rúmen está diteramente relacionada com a digestibilidade da dieta, uma vez que dietas mais digestíveis elevam a presença de protozoários no rúmen. Esses eucaritos apresentam papel fundamental na modulação da taxa de fermentação ruminal, favorecendo o equilíbrio no ecossistema ruminal e, evitando disfunções metabólicas (Lima et al., 2012).

Segundo Reis (2015) a dieta, é um dos principais fatores reguladores da concentração e a distribuição dos gêneros de protozoários ruminais. Modificações na dieta impõe ao animal um período de transição na população microbiana, com mudanças nas proporções entre as diferentes espécies, promovendo um novo equilíbrio e prevalecendo as espécies que melhor se adaptam a nova dieta.

O sorgo e o milho apresentam características importantes na dieta dos ruminantes por serem alimentos ricos em amido, que constitui a principal fonte energética nos alimentos concentrados para alimentação animal. O processamento desses grãos melhora a digestibilidade ruminal do amido, aumentando a fermentação ruminal e a disponibilidade de energia para a microbiota ruminal, elevando a população de ciliados no rúmen (Oliveira et al., 2015).

Os estudos em microbiologia ruminal estão constantemente voltados às bactérias, porém os protozoários podem utilizar uma fração significativa dos carboidratos solúveis, amidos e carboidratos da parede celular para o crescimento, além de estarem associados à maior reciclagem de nitrogênio microbiano no rúmen e à redução no suprimento de aminoácidos ao intestino (Ivan et al., 2001).

Novas pesquisas sobre a modulação da microbiota ruminal, principalmente sobre a população de protozoários, representam uma demanda mundial, portanto visando contribuir para novos conhecimentos, objetivou-se com a presente pesquisa caracterizar as populações de protozoários do rúmen de ovinos alimentados com milho e sorgo submetidos a diferentes tipos de processamentos.

\section{MATERIAL E MÉTODOS}

O experimento foi conduzido no setor de Ovinocultura do Instituto Federal Goiano localizado no município de Ceres-GO (Latitude: $15^{\circ} 18^{\prime} 30^{\prime \prime} \mathrm{S}$, Longitude: $49^{\circ} 35^{\prime} 54^{\prime \prime}$ W, Altitude: 571m).

Tabela I. Composição das dietas experimentais, expressa na matéria seca (\% MS) (Composition of experimental diets, expressed in dry matter content (\% DM)).

\begin{tabular}{|c|c|c|c|c|}
\hline \multirow{2}{*}{ Variável } & \multicolumn{4}{|c|}{ Tratamentos } \\
\hline & MGS & MGR & SGS & SGR \\
\hline \multicolumn{5}{|l|}{ Ingredientes (\%) } \\
\hline Feno & 40,00 & 40,00 & 40,00 & 40,00 \\
\hline Milho Grão Seco & 43,39 & - & - & - \\
\hline Milho Grão Reidratado & - & 42,74 & - & - \\
\hline Sorgo Grão Seco & - & - & 45,29 & - \\
\hline Sorgo Grão Reidratado & - & - & - & 44,79 \\
\hline Farelo de soja & 14,85 & 15,48 & 12,95 & 13,45 \\
\hline Ureia & 0,30 & 0,30 & 0,30 & 0,30 \\
\hline Calcário calcítico & 0,20 & 0,20 & 0,20 & 0,20 \\
\hline Suplemento mineral e vitamínico ${ }^{1}$ & 1,20 & 1,20 & 1,20 & 1,20 \\
\hline Monensina sódica & 0,06 & 0,06 & 0,06 & 0,06 \\
\hline \multicolumn{5}{|l|}{ Composição Química (\%) } \\
\hline Matéria Seca (MS) & 88,76 & 74,72 & 88,82 & 75,92 \\
\hline Nutrientes digestíveis totais (NDT) & 77,12 & 76,76 & 76,88 & 75,98 \\
\hline Proteína bruta (PB) & 15,92 & 16,16 & 15,26 & 15,68 \\
\hline Extrato etéreo (EE) & 2,24 & 2,36 & 1,76 & 1,94 \\
\hline Fibra detergente neutro (FDN) & 36,04 & 37,18 & 38,26 & 38,02 \\
\hline Fibra detergente ácido (FDA) & 18,06 & 18,96 & 19,26 & 19,56 \\
\hline Matéria mineral (MM) & 3,16 & 3,28 & 2,58 & 3,10 \\
\hline Cálcio (Ca) & 0,39 & 0,49 & 0,39 & 0,44 \\
\hline Fósforo (P) & 0,30 & 0,34 & 0,29 & 0,32 \\
\hline $\mathrm{pH}$ & 5,23 & 4,63 & 5,21 & 4,58 \\
\hline
\end{tabular}

${ }^{1}$ Composição por kg do produto: Vitamina A 50.000 UI, Vitamina E 312 UI, Ferro 1.000 mg, Manganês 3.000 mg, Zinco 5.000 mg, lodo 60 $\mathrm{mg}$, Cobalto $80 \mathrm{mg}$, Selênio $10 \mathrm{mg}$, Magnésio $10 \mathrm{~g}$, Enxofre $12 \mathrm{~g}$, Cálcio $140 \mathrm{~g}$, Fósforo $65 \mathrm{~g}$, Umidade $10 \mathrm{~g}$, Sódio $130 \mathrm{~g}$, Flúor 650 mg. 
Foram utilizadas 28 borregas da raça Santa Inês com idade média de nove meses, peso médio inicial de $36 \mathrm{Kg}$, confinadas em baias coletivas, com comedouros e de bebedouros em uma área de $15 \mathrm{~m}^{2}$, distribuídas aleatoriamente em quatro lotes com sete animais, sendo cada animal uma repetição.

As borregas receberam dietas isoenergéticas e isoprotéicas com relação volumoso: concentrado de 40:60 na matéria seca (MS), sendo os tratamentos: Milho Grão Seco (MGS), Milho Grão Reidratado (MGR), Sorgo Grão Seco (SGS) e Sorgo Grão Reidratado (SGR), com dietas balanceadas (Tabela I) de acordo com o NRC (1985). O milho e o sorgo foram moídos em moinho do tipo martelo com peneira de crivo $3 \mathrm{~mm}$. O mesmo procedimento de moagem foi feito em outra fração da mesma partida dos grãos, e posteriormente reidratado, estimando para umidade de 35\%, e ensilados em silo trincheira por 90 dias.

O concentrado foi composto pelos alimentos testados (MGS, MGR, SGS e SGR), além de farelo de soja, ureia, suplemento mineral para ovinos, calcário calcítico e monensina sódica. O volumoso da dieta foi o feno de capim bermudas (Cynodom dactylon). $\mathrm{O}$ fornecimento do alimento foi realizado duas vezes ao dia, 07:00 e 16:00 horas, na forma de dieta total, com água sempre à disposição dos animais. O experimento teve duração de 60 dias, sendo os 15 primeiros dias destinados à adaptação dos animais a dietas e instalações, totalizando 45 dias de ensaio experimental.

A coleta de fluido ruminal foi realizada no período de oito às 11 horas da manhã, no último dia do confinamento. Após prévio jejum aproximado de oito a 12 horas, foi realizado em uma área de aproximadamente cinco $\mathrm{cm}^{2}$, localizada na parte ventral do abdome esquerdo, abaixo da fossa paralombar e cranialmente à articulação do joelho, a tricotomia e a assepsia, com solução de Polivinilpirrolidona-Iodo (Iodo-PVP) (1\%) (Dirksen, 1993). Foram puncionados aproximadamente $10 \mathrm{~mL}$ de fluido ruminal, com o auxílio de cateter humano (Solidor $®, 14,2$, Bio Med health Care Products, Haryana- India), acoplado a seringas estéreis. Todos os procedimentos realizados foram submetidos e aprovados pela Comissão de Ética no Uso de Animais do Instituto Federal Goiano (CEUA - IF Goiano), com número de Protocolo: $017 / 2014$.

As amostras foram transportadas em caixas isotérmicas e armazenadas por no máximo uma hora em tubos de ensaio vedados e estéreis. As análises microbiológicas foram realizadas no laboratório de microbiologia do Instituto Federal Goiano (Campus Ceres). A análise macroscópica do líquido coletado foi realizada imediatamente após a coleta, em um tubo de vidro contendo cinco mL do suco amostrado, para avaliação da cor, odor e viscosidade (Dirksen, 1993). Para avaliação da atividade microbiana no rúmen utilizou o teste de redução do azul de metileno na concentração 0,03\% (potencial redox). $\mathrm{O}$ pH do líquido ruminal foi estimado, utilizando um potenciômetro digital (Dirksen, 1993).

Amostras do fluido ruminal coletado foram filtradas em camadas de gazes e uma alíquota de um $\mathrm{mL}$ do suco ruminal de cada animal, foi diluída em nove $\mathrm{mL}$ de solução de formaldeído a $10 \%$, para a conservação das estruturas micro morfológicas dos protozoários, em seguida, quando necessário eram realizadas diluições decimais em solução salina (Dehority, 1984).

Para a quantificação de protozoários pequenos, médios e grandes, foi inoculado um $\mathrm{mL}$ da solução acima em câmara de Sedgwick Rafter, para visualização e quantificação sob a luz do microscópio óptico, utilizando a objetiva de 10x (Dehority, 1984). Para identificação dos gêneros desses microrganismos ruminais, foi utilizada uma gota da diluição $10^{-1}$ descrita anteriormente juntamente com uma gota de azul de metileno em lâmina de microscopia. Nessas lâminas, foram acopladas lamínulas para visualização, em microscópio óptico, utilizando-se objetiva de 10x, das microestruturas dos protozoários e posterior classificação de acordo com a chave descrita por (Dehority, 1984).

Para as variáveis estudadas foi adotado um delineamento inteiramente ao acaso. Os resultados foram submetidos à análise estatística pelo software R (R Development Core Team, 2013), ao comparar a quantificação de grupos de protozoários pelo teste de Kruskall-wallis $(\alpha=0,05)$. As taxas de detecção dos gêneros identificados foram comparadas pelo teste do Qui-quadrado, a 5\% de probabilidade, pelo software R (R Development Core Team, 2013).

\section{RESULTADOS E DISCUSSÕES}

\section{EXAMES MACROSCÓPICOS E FÍSICO-QUÍMICOS DO FLUIDO} RUMINAL

$\mathrm{O} \mathrm{pH}$ do fluido ruminal apresentou diferença significativa $(p<0,05)$ entre os quatros tratamentos (Tabela II). Sendo observado $\mathrm{pH}$ mais ácido para ovinos alimentados com MGR $(6,24)$ e menos ácido para MGS e SGS (6,75 e 6,61, respectivamente). As alterações no $\mathrm{pH}$ ruminal podem estar relacionadas com a alimentação ingerida, características inerentes aos animais, como taxa de ingestão alimentar, salivação, taxa de fermentação e concentrações de seus 
produtos finais (Russel \& Rychlik, 1994; Martinele et al., 2008).

As borregas alimentadas com dietas contendo os tratamentos MGR e SGR, apresentaram pH inferior aos demais, podendo notar uma tendência de $\mathrm{pH}$ mais ácido quando o grão passa pelo processo de reidratação e ensilagem. Este resultado corrobora com estudo de Santra et al. (2003), onde dietas que não estimulam a mastigação reduzem a produção de saliva, resultado em diminuição acentuada do $\mathrm{pH}$ ruminal.

Acredita-se que o $\mathrm{pH}$ ruminal dos ovinos poderia estar ainda mais baixo durante o manejo normal, uma vez que estavam sob jejum de aproximadamente 12 horas. Segundo Vieira (2015), animais nessa fase podem estar sob efeito tamponante da saliva.

Oliveira et al. (2015) estudaram o pH ruminal de cordeiros alimentados com milho grão seco moído e silagem de grão úmido de milho, observaram que não ocorreu alterações entre os processamentos, apresentando médias de 6,62 e 6,63, respectivamente.

Para as características do líquido ruminal, pôdese observar que todas as amostras apresentaram coloração verde oliva. O resultado apontado já era esperado, uma vez que todos os animais estavam recebendo fonte volumosa na dieta. Dirksen (1993) relatou que a cor do suco ruminal pode variar de acordo com a alimentação ofertada aos animais.

Borges et al. (2011) estudaram o líquido ruminal de ovinos confinados submetidos a dieta básica de feno de Uroclhoa (Brachiaria) decumbens e suplementadas com níveis crescente de mistura mineral-energético-proteico e encontraram coloração verde variando a tonalidade (amarelado, acastanhado e oliva).

O odor do fluido ruminal foi aromático para todas as amostras. A viscosidade foi levemente espessa e a redução do azul de metileno apresentou-se acima de três minutos para a maioria dos ovinos, porém foi observado em alguns ovinos microbiota mais ativa, com PRAM menor que três minutos para um ovino do tratamento MGS, um do MGR e dois do SGS. Confor- me indica Dirksen (1981), quando a atividade microbiana está elevada ocorre à descoloração do azul de metileno em 3 minutos, quando apresenta atividade moderada a descoloração requer um tempo maior de 6 minutos, indicando assim que a atividade do fluido ruminal diminuiu.

\section{QUANTIFICAÇÃO DA POPULAÇÃO PROTOZOÁRICA NO RÚMEN}

A quantidade total de protozoários e as proporções de médios e grandes presentes no ambiente ruminal não foram influenciadas $(\mathrm{P}>0,05)$ pelos tratamentos. Porém a quantidade de protozoários pequenos (ilustrado na Figura 1) foi significativamente superior $(\mathrm{P}<0,05)$ nos tratamentos constituídos por SGR $\left(9,51 \times 10^{5}\right)$ e inferior com MGS $\left(6,21 \times 10^{5}\right)$ (Tabela III).

Segundo indicaram Borges et al. (2011) a quantidade de pequenos protozoários no rúmen prevalece sobre os médios e os grandes na maioria dos casos, apresentado frequência igual ou superior a $90 \%$, o que sugere o predomínio do gênero Entodinium.

Os dados indicam que o SGR favorece o crescimento da população de pequenos protozoários no rúmen, quando comparado ao MGS. A concentração total de protozoários detectados está dentro dos valores normais que correspondem a $10^{4}$ a $10^{6}$ protozoários / $\mathrm{mL}$ de conteúdo ruminal (Kamra, 2005).

Avaliando os diferentes tipos de processamento do grão de milho (inteiro, moído e silagem de grão úmido) na alimentação de cordeiros confinados, pesquisadores afirmaram que o processamento do grão favorece o crescimento populacional de protozoários no rúmen, devido a maior disponibilidade do amido para a microbiota ruminal (Oliveira et al., 2015).

Hook et al. (2011) ao avaliarem a densidade de protozoários no rúmen de vacas leiteira não lactantes alimentadas com dietas contendo alto nível de concentrado ou somente com volumoso, relataram aumento no número de protozoários no conteúdo ruminal quando alimentados com dieta rica em grãos. Segundo Solórzano (2012) a quantidade de protozoários pode ser relativamente baixa em animais re-

Tabela II. Análise físico-química do conteúdo ruminal de borregas alimentadas com diferentes tipos de processamento de milho e sorgo (Physical-chemical analysis of ruminal content of lambs fed with different types of processing of maize and sorghum).

\begin{tabular}{lccccc}
\hline Físico-química & MGS & MGR & SGS & SGR & CV (\%) \\
\hline PRAM & $>3$ & $>3$ & $>3$ & $>3$ & - \\
$\mathrm{pH}$ & $6,75^{\mathrm{b}}$ & $6,24^{\mathrm{a}}$ & $6,61^{\mathrm{b}}$ & $6,42^{\mathrm{ab}}$ & 3,6
\end{tabular}

Nota: Letras iguais na mesma linha não diferem entre si pelo teste de Tukey, a 5\% de probabilidade. CV (\%) - coeficiente de variação; PRAM - potencial de redução do azul de metileno. * MGS: milho grão seco, MGR: milho grão reidratado, SGS: sorgo grão seco, SGR: sorgo grão reidratado. 


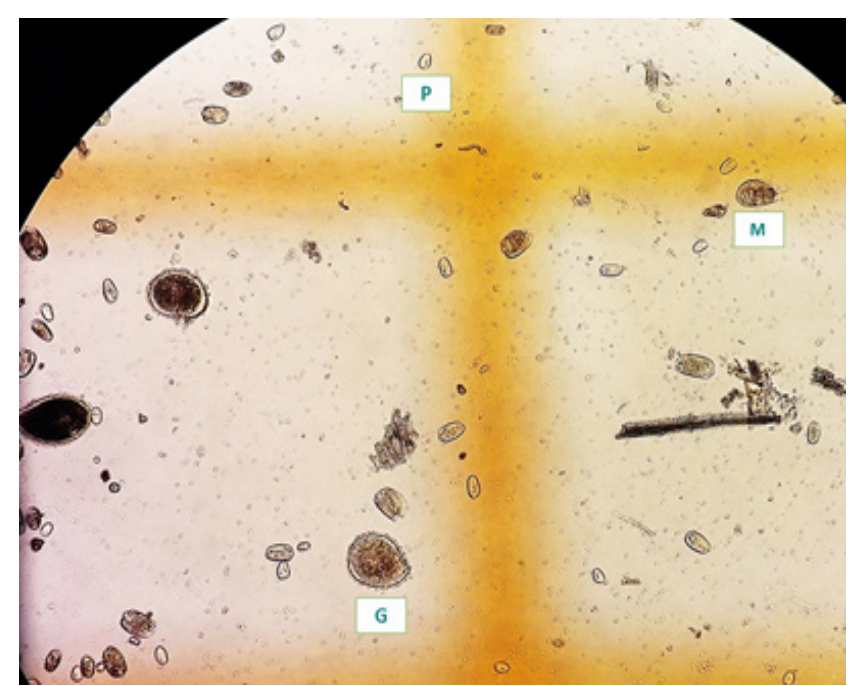

Figura 1. Protozoários ruminais de borregas Santa Inês; P: pequeno, M: médio e G: grande (Ruminal protozoa of lambs Santa Inês; P: small; M: medium and G: large).

cebendo dietas exclusivas de forragens, e maior nas misturas de forragens e grãos.

Segundo Wlodarski (2017) os protozoários realizam o engolfamento das bactérias com objetivo de utilizar os aminoácidos e ácidos nucleicos presentes. Dietas com maior concentração de grãos torna esse processo mais intenso e simplificado do que comparado com dietas ricas em forragens, pois as bactérias constituem sítios de aderência nos carboidratos e dificultam o engolfamento pelos protozoários, que diminui a quantidade de protozoários no rúmen.

\section{IDENTIFICAÇÃO DOS GÊNEROS DE PROTOZOÁRIOS NO RÚMEN}

A distribuição dos gêneros de protozoários identificados pode ser observada na Tabela IV. Foram identificados nove gêneros de ciliados das famílias Blepharocorythidae (Charonina spp.), Isotrichidae (Isotricha spp., Dasytricha spp.) e Ophryoscolecidae (Entodinium spp., Diplodinium spp., Diploplastron spp., Eremoplastron spp., Eudiplodinium spp. e Polyplastron spp.)

$\mathrm{O}$ gênero Entodinium predominou dentre os protozoários para todos os tratamentos testados (MGS, MGR, SGS e SGR), que variou de 90,36 a $92,52 \%$ da concentração total, concordando com a citação de Dehority (1991) de que, em geral, cerca de $90 \%$ da fauna de ruminantes é constituída de entodínios. No entanto a população de Entodinium ocorreu em maior frequência $(\mathrm{p}<0,05)$ nos animais alimentados com SGS em relação com MGS.

Segundo Ogimoto \& Imai (1981) essa predominância do gênero Entodinium pode ocorrer devido ao fato

Tabela III. Médias de protozoários por mililitro de fluido ruminal e porcentagem de protozoários pequenos, médios e grandes de borregas submetidas a diferentes dietas (Averages of protozoa per millilitre of rumen fluid and percentage of protozoa small, medium and large ewe lambs subjected the different diets).

\begin{tabular}{|c|c|c|c|c|c|c|c|c|}
\hline \multirow{3}{*}{ Tratamentos } & \multicolumn{8}{|c|}{ Protozoários $/ \mathrm{mL}$} \\
\hline & \multicolumn{2}{|c|}{ Pequeno } & \multicolumn{2}{|c|}{ Médio } & \multicolumn{2}{|c|}{ Grande } & \multicolumn{2}{|c|}{ Total } \\
\hline & $\mathrm{N}$ & $\%$ & $\mathrm{~N}$ & $\%$ & $\mathrm{~N}$ & $\%$ & $\mathrm{~N}$ & $\%$ \\
\hline MGS & $6,21 \times 10^{5 b}$ & $48,2 \%$ & $6,41 \times 10^{5}$ & $49,8 \%$ & $2,63 \times 10^{4}$ & $2,0 \%$ & $1,29 \times 10^{6}$ & $100 \%$ \\
\hline MGR & $6,69 \times 10^{5 a b}$ & $48,5 \%$ & $6,91 \times 10^{5}$ & $50,3 \%$ & $1,71 \times 10^{4}$ & $1,2 \%$ & $1,38 \times 10^{6}$ & $100 \%$ \\
\hline SGS & $7,51 \times 10^{5 a b}$ & $57,5 \%$ & $5,37 \times 10^{5}$ & $41,1 \%$ & $1,79 \times 10^{4}$ & $1,4 \%$ & $1,31 \times 10^{6}$ & $100 \%$ \\
\hline SGR & $9,51 \times 10^{5 a}$ & $51,7 \%$ & $8,48 \times 10^{5}$ & $46,2 \%$ & $3,83 \times 10^{4}$ & $2,1 \%$ & $1,84 \times 10^{6}$ & $100 \%$ \\
\hline
\end{tabular}

Nota: Letras minúsculas diferentes na mesma coluna indicam diferença estatística pelo teste não paramétrico de Kruskal-Wallis ( $\alpha=5 \%)$.

Tabela IV. Distribuição dos gêneros de protozoários ruminais de borregas submetidas a diferentes dietas. (Distribution of genera of ruminal protozoa of ewe lambs subjected to different diets).

\begin{tabular}{|c|c|c|c|c|c|c|c|c|c|}
\hline \multirow{2}{*}{ Gêneros } & \multicolumn{9}{|c|}{ Tratamentos } \\
\hline & MGS & $\%$ & MGR & $\%$ & SGS & $\%$ & SGR & $\%$ & Total \\
\hline Charonina spp & $174^{\mathrm{Ab}}$ & 4,29 & $270^{A c}$ & 3,99 & $144^{\mathrm{Bc}}$ & 1,68 & $97^{\mathrm{Bd}}$ & 1,58 & 685 \\
\hline Dasytricha spp. & $8^{\mathrm{Ad}}$ & 0,20 & $18^{\mathrm{Ae}}$ & 0,26 & $22 \mathrm{Ae}$ & 0,26 & $45^{\mathrm{Af}}$ & 0,73 & 93 \\
\hline Diplodinium spp. & $120^{\mathrm{Bc}}$ & 2,97 & $315^{\mathrm{Ab}}$ & 4,65 & $290^{\mathrm{Bb}}$ & 3,38 & $202^{\mathrm{Bb}}$ & 3,29 & 927 \\
\hline Diploplastron spp. & $5^{\mathrm{Bd}}$ & 0,12 & $0^{B f}$ & 0,00 & $105^{\mathrm{Ad}}$ & 1,22 & $74 \mathrm{Ae}$ & 1,21 & 184 \\
\hline Entodinium spp. & $3738 \mathrm{Ca}$ & 92,25 & $6121 \mathrm{Ba}$ & 90,36 & 7938 Aa & 92,52 & $5610^{\mathrm{Ca}}$ & 91,46 & 23407 \\
\hline Eremoplastron spp. & $0^{\mathrm{Ad}}$ & 0,0 & $4^{\mathrm{Af}}$ & 0,06 & $1^{\text {Af }}$ & 0,01 & $0^{\mathrm{Ag}}$ & 0,00 & 5 \\
\hline Eudiplodinium spp. & $2^{\mathrm{Ad}}$ & 0,05 & $0^{\text {Af }}$ & 0,00 & $0^{A f}$ & 0,00 & $1^{\mathrm{Ag}}$ & 0,02 & 3 \\
\hline Isotricha spp. & $5^{\mathrm{cd}}$ & 0,12 & $42^{\mathrm{Bd}}$ & 0,62 & $78^{\mathrm{Be}}$ & 0,91 & $105^{\mathrm{Ac}}$ & 1,71 & 230 \\
\hline Polyplastron spp. & $0^{\mathrm{Ad}}$ & 0,0 & 4 Af & 0,06 & $2^{\text {Af }}$ & 0,02 & $0^{\mathrm{Ag}}$ & 0,00 & 6 \\
\hline Total & 4052 & 100 & 6774 & 100 & 8580 & 100 & 6134 & 100 & 25540 \\
\hline
\end{tabular}

Frequências com mesmas letras, maiúsculas nas linhas e minúsculas na coluna, não diferem entre si, comparadas estatisticamente pelo teste de Qui-quadrado a $5 \%$ de probabilidade. 
de que este gênero apresenta várias espécies com ampla distribuição e ocorrência dentre vários ruminantes domésticos e selvagens. A maior composição de Entodinium também pode estar associada à velocidade de reprodução deste gênero (Matos et al, 2008).

Oliveira et al. (2015) evidenciaram que o gênero Entodinium foi o mais frequente para cordeiros confinados alimentados com grão de milho (inteiro, moído e silagem de grão úmido), constituído $78,20 \%$ da população total. Já Carvalho et al. (2011) observaram que a substituição do milho pela casca de soja reduz a população de protozoários do gênero Entodinium no conteúdo ruminal de caprinos, sendo que a diminuição pode estar associada à redução no teor de nutrientes digestíveis totais e aumento no teor de fibra em detergente neutro. Embora a concentração de Entodinium spp. tenha reduzido com aumento no nível da casca de soja, este gênero predominou em todas as dietas, com variação de 70,39 a $86,28 \%$ da concentração total.

\section{CONCLUSÕES}

A concentração de médios e grandes protozoários não é influenciada pelo tratamento de grãos em dieta mista, no entanto a população de pequenos protozoários é superior quando a fonte de concentrado é o sorgo grão reidratado e ensilado e inferior em dietas com fonte concentrada constituída por milho grão seco. O gênero Entodinium é o mais frequentes dentre os protozoários para todos os tratamentos avaliados.

O processamento do grão de sorgo reidratado favorece o crescimento da população de pequenos protozoários no rúmen e a população do gênero Entodinium é mais frequente nos ovinos alimentados com sorgo grão seco.

\section{AGRADECIMENTOS}

Ao Instituto Federal Goiano - Campus Ceres pelo apoio e pela disponibilidade dos animais. À Agrocria Nutrição Animal e Sementes pelo financiamento do sorgo.

\section{BIBLIOGRAFIA}

Borges, NC, Orsine, GF, Silva, LAF, Bernardes, KM, Martins, MEP \& Fioravanti, MC, S 2011, 'Parâmetros físico-químicos e microbiológicos do fluido ruminal de ovinos confinados submetidos a crescentes níveis de mistura mineral energético-proteica', Ciência Animal Brasileira, vol. 12, no. 3, pp. 392-399.

Carvalho, LFPB, Amorim, GL, Matos, DS, Batista, AMV, Moraes, ACA \& Cabral, AMD 2011 , 'Protozoários do rúmen de caprinos submetidos à dieta com casca de soja', Revista Brasileira de Saúde e Produção Animal, vol. 12, no. 1, pp. 244-253.

Dehority, BA 1984, 'Evaluation of subsampling and fixation procedures used for counting rumen protozoa', Applied Environmental Microbiology, vol. 48, no. 1, pp. 182-185.
Dehority, BA 1991, 'Rumen microbiology', Wooster, USA: OARDC/ OSU, $87 \mathrm{pp}$.

Dirksen, G 1981, Indigestiones en el bovino, Schnetztor-Verlag, Konstanz, 76 pp.

Dirksen, G 1993, 'Sistema digestivo', in: Dirksen, G, et al. (eds), Rosenberg: Exame Clínico dos Bovinos, Guanabara-Koogan: Rio de janeiro, pp. 167-169.

Hook, SE, Steele, MA, Northwood, KS, Wright, ADG \& Mcbride, BW 2011, 'Impact of high-concentrate feeding and low ruminal pH on methanogens and protozoa in the rumen of dairy cows', Microbial ecology, vol. 62, no. 1, pp. 94-105.

Ivan, M, Mir, PS, Koenig, KM, Rode, LM, Neil, L, Entz, Z \& Mir, Z 2001, 'Effects of dietary sunflower seed oil on rumen protozoa population and tissue concentration of conjugated linoleic acid in sheep', Small Ruminant Research, vol. 41, no. 3, pp. 221-227.

Kamra, DN 2005, Rumen Microbial Ecosystem, Current Science, vol. 89 , pp. 35-125.

Lima, ME, Vendramin, L, Hoffmann, DAC, Lisboa, FP, Gallina, T, Rabassa, VR, Schwegler, E \& Corrêa, MN 2012, 'Alterações na população de protozoários ruminais, quantificados a partir da adaptação da técnica de Dehority, de ovinos submetidos a uma dieta de confinamento', Acta Scientiae Veterinariae, vol. 40, no. 1, pp. 1-6.

Martinele, I, Siqueira, ICVC, D'Agosto, M 2008. 'Protozoários ciliados no rúmen de bovinos alimentados com dietas de capim-elefante e com dois níveis de concentrado', Revista Brasileira de Saúde e Produção Animal, vol. 9, no. 1, pp. 74-81.

Matos, DS, Guim, A, Batista, AMV, Santos, MVF, Correa, IM, Santos, GRA \& Lopes, CRA 2008. 'População de protozoários ciliados no rúmen de ovinos criados na caatinga de Pernambuco', Revista Brasileira de Saúde e Produção Animal, vol. 9, no. 2, pp. 270-279.

National Research Council 1985, Nutrient requirements of sheep, National Academy Press. Ed. Washington, DC.

Ogimoto, K \& Imai, S 1981, 'Atlas of rumen microbiology', Tokyo: Japan Scientific Societies, $231 \mathrm{pp}$.

Oliveira, LS, Manzo, MR, Carvalho, RF, Pesce, DMC, Silva, SL, Nogueira, FJCM, Gallo, SB \& Leme, PR 2015, 'Processamento do milho grão sobre desempenho e saúde ruminal de cordeiro', Ciência Rural, vol. 45, no. 7, pp. 1292-1298.

R Development Core Team 2013, R: A language and environment for statistical computing, R Foundation for Statistical Computing, Austria, ISBN 3-900051-07-0, acesso em 15 de Janeiro 2017, <http:// www.R-project.org>.

Reis, CC 2015, 'Protozoários ciliados no rúmen de bovinos Nelore e Cruzados Nelore x Europeu sob diferentes sistemas de alimentação', Dissertação de Mestrado, Universidade Tecnológica Federal do Paraná, Dois Vizinhos.

Russell, JB \& Rychlik, JL 1994, 'Factors that alter rumen microbial ecology', Science, vol. 292, no. 5519, pp. 1119-1122.

Santra, A, Chaturvedi, OH \& Tripathi, MK 2003,'Effect of dietary sodium bicarbonate supplementation on fermentation characteristics and ciliate protozoal populations in rumen of lambs', Small Ruminant Research, vol. 47, no. 3, pp. 203-212.

Solórzano, LAR 2012, 'Efeito de fontes energéticas sobre a fermentação ruminal, produção de metano determinada pela técnica de gás traçador SF6, digestibilidade aparente total e excreção de nutrientes em bovinos', Dissertação de Mestrado, Universidade de São Paulo, Pirassununga.

Vieira, EA, Abrão, FO, Ribeiro, ICO, Nigri, ACA, Silva, KF, Careli, RT, Geraseev, LC \& Duarte, ER 2015, 'Bastonetes Gram-negativos 
aeróbios e anaeróbios facultativos no fluido ruminal de bovinos de corte alimentados em pastagem lignificada e em novilhos com acidose ruminal', Pesquisa Veterinária Brasileira, vol. 35, no. 9, pp. 1-6.
Wlodarski, L 2017, 'Determinação e quantificação de protozoários ciliados e bactérias do rúmen de bovinos em pastagens temperadas e tropicais', Dissertação de Mestrado, Universidade Tecnológica Federal do Paraná, Campus Dois Vizinhos. 